\section{The Swallow Physiology Change of Balloon Dilatation on Pharyngeal Dysphagia}

\author{
Ping Wan ${ }^{1 \#}$, Xuhui Chen ${ }^{2 *}$, Mingwen $\mathrm{Yu}^{1}$, Xing $\mathrm{Wei}^{3}$, Li \\ Huang ${ }^{4}$, Shuangjing $X u^{1}$, Yabin $\mathrm{Yu}^{2}$, Rui $\mathrm{Qi}^{4^{*}}$ and Ruiying \\ Ding $^{5^{*}}$
}

${ }^{1}$ School of Rehabilitation Medicine, Shanghai University of Traditional Chinese Medicine, Shanghai, China

${ }^{2}$ Department of Otorhinolaryngology, Yueyang Hospital of Integrated Traditional Chinese and Western Medicine affiliated to Shanghai University of Traditional Chinese Medicine, Shanghai, China

${ }^{3}$ Department of Neurology, Yueyang Hospital of Integrated Traditional Chinese and Western Medicine affiliated to Shanghai University of Traditional Chinese Medicine, Shanghai, China

${ }^{4}$ Department of Rehabilitation, Yueyang Hospital of Integrated Traditional Chinese and Western Medicine affiliated to Shanghai University of Traditional Chinese Medicine, Shanghai, China

${ }^{5}$ Department of Communication Sciences and Disorders, Elmhurst College, Illinois, USA

\# - These authors contributed equally.
Each session lasted 30 minutes, with eight dilatations per session, three sessions per week for two weeks.

Results

There was significant reduction in pharyngeal residue pre-versus post-balloon dilatation $(p=0.002)$. There were also significant improvements in cricopharyngeal muscle achalasia $(p=0.004)$ and weak pharyngeal contraction $(p=0.008)$ pre-versus post-treatment. Eight out of the 12 patients made significant progress toward oral feeding $(66.67 \%)$.

\section{Conclusion}

Our study found cricopharyngeal muscle achalasia and weak pharyngeal contraction were closely related to pyriform sinus residue in pharyngeal dysphagia. Balloon dilatation eliminated cricopharyngeal muscle achalasia and weak pharyngeal contraction and significantly reduced pyriform sinus residue in 8 of the 12 patients. Balloon dilatation seems to have no effect on the reduction of pyriform sinus residue when the cause was either from discoordination of swallowing subsystems in the case of infarction of the left pontocerebellar trigone or loss of large bulk of tongue tissue in the case of cancer treatment.

Keywords: Balloon dilatation; Deglutition disorder; Pharyngeal dysphagia; Pyriform sinus residue; Rehabilitation

Level of Evidence: 4 Case Series

\section{Introduction}

One of the most common signs of pharyngeal dysphagia is Pyriform Sinus Residue (PSR), which is the retention of more than a thin mucosal coating of material in the pyriform sinuses after swallowing [1]. Patients with PSR have inefficient swallow and often present with post-swallow aspiration and aspiration pneumonia, which can be life threatening.

PSR could be resulted from various swallow physiology changes including Cricopharyngeal Muscle Achalasia (CMA) in which case cricopharyngeal muscle could not relax during swallowing [2], incomplete laryngeal elevation, and/or impaired pharyngeal driving force [3]. These conditions can be seen in strokes of various locations, including subcortical and supratentorial, lateral medullary and cerebellum [4-6]. PSR was also found in head and neck cancer patients [7], most commonly caused by reduced tongue mass and limited tongue mobility that was associated with total or partial glossectomy with or without reconstruction [8,9], and radiation-induced esophageal strictures as well [10]. Radiation therapy post nasopharyngeal carcinoma is associated with impaired pharyngeal contraction, which can lead to PSR [11].

Several methods have been used to treat Cricopharyngeal Muscle Achalasia (CMA), such as rigid dilators [12] or Botox injection planning [13], but as one of more convenient, less invasive methods, Balloon Dilatation (BD) has been used in more studies to treat cricopharyngeal dysfunction and pharyngo-upper esophageal strictures $[2,6,10,14-16]$. It has been shown to improve UES relaxation and pharyngeal contraction amplitudes $[6,14,15]$. Recannulation through serial dilation is possible even in cases of complete or near-complete cricopharyngeus stricture in head and neck cancer patients after radiation therapy $[10,15]$. Therefore, it is hypothesized that $\mathrm{BD}$ will be effective in reducing PSR. The current study included patients with various swallow physiology changes associated with different medical 
Citation: Wan P, Chen X, Yu M, Wei X, Huang L, et al. (2017) The Swallow Physiology Change of Balloon Dilatation on Pharyngeal Dysphagia. J Otolaryng Head Neck Surg 3: 014.

diagnoses, it allowed us to explore the effectiveness spectrum of BD in reducing PSR in a variety of patients.

\section{Materials and Methods}

\section{Participants}

Between March 2009 and July 2015, 117 patients with dysphagia were seen in the Department of Rehabilitation and Department of Otolaryngology at Yueyang Hospital. Their medical records were retrospectively reviewed and 12 patients who satisfied the following inclusion criteria were studied.

1. Had both fiberoptic Endoscopic Evaluation of Swallowing (FEES) and Video Fluoroscopic Swallowing Assessment (VFSS) pre-and post-BD. All swallowing difficulties were observed in the initial imaging studies of FEES and VFSS. The initial FEES and VFSS studies were performed from 5 days to one year post onset of the clinical dysphagia symptoms, and BD was performed three days after the initial FEES and VFSS. A second FEES and VFSS were performed 24 hours post the final BD session

2. Confirmed severe PSR (residue filling $>50 \%$ of the height of the pyriform sinus) [17]

3. Received BD treatment

The study was approved by the local ethics committee and informed consent for BD and future research study involving BD was obtained from all participants. The subjects included 10 males and 2 females aged from 40 to 71 years (mean \pm SD, $58 \pm 9$ years) with diagnoses of: brain infarction (8 patients), nasopharyngeal carcinoma post-radiation ( 2 patients), oral cancer post partial glossectomy (1 patient), and motor neuron disease (1 patient). In the 8 patients with infarction, contrast-enhanced Computed Tomography (CT) or Magnetic Resonance Imaging (MRI) was used to identify the location of fresh infarcts [18]; these locations are shown in table 1. For 6 patients it was their first stroke and for the other 2 patients their second stroke.
Even though traditional swallow therapy was initiated immediate after stroke when swallow difficulty was noted, dysphagia persisted until the time BD was attempted. The patients' feeding and swallowing function were evaluated with the Functional Oral Intake Scale (FOIS) prior to the initiation of BD [19]. All patients were assessed to be either level 1 (nothing by mouth) or level 2 (tube dependent with minimal attempts at liquid intake) and received nutrition through a nasogastric tube (patients no. 1-11) or gastrostomy tube (no.12) at the time of the study.

\section{Imaging studies of swallow evaluation}

Every patient was assessed with FEES and VFSS almost concurrently both in the pre-and post-treatment sessions. FEES were performed first followed by VFSS 30 minutes later. First, the patient was instructed to make a forceful "eee" sound in a pharyngeal squeeze maneuver. Fuller et al., and Rodriguez $[20,21]$ both reported that the pharyngeal squeeze maneuver is a valid surrogate measure of pharyngeal motor integrity and has high reliability. Next, patients were instructed to swallow one $3 \mathrm{ml}$ spoonful of yogurt. The following data were collected from the FEES study:

1. Weak Pharyngeal Contraction (WPC). If the patient could not use the pharyngeal squeeze maneuver to make sound because of muscle problem, WPC was indicated

2. The ratio of PSR bilaterally (residue area/pyriform sinus area) [17]

In VFSS, each patient completed one $3 \mathrm{ml}$ swallow in the lateral view and the value for the following measurement was obtained using Neusoft software (PACS system, China) [22-24]:

1. The ratio of PSR (residue area/pyriform sinus area)

2. Pharyngeal Delay Time (PDT, the duration between when the head of the bolus reaches the lower rim of the mandible and the onset of laryngeal elevation). Normal range is 0 to $0.2 \mathrm{~s}$

\begin{tabular}{|c|c|c|c|c|c|}
\hline Patient No. & Gender & Age & Diagnosis & Frequency & Location of Cerebral Infarction (CT/MRI) \\
\hline 1 & Male & 54 & $\begin{array}{l}\text { Acute Ischemic Cerebral Infarction } \\
\text { (AICI) }\end{array}$ & First time & Lacunar infarction of bilateral basal ganglia \\
\hline 2 & Male & 70 & AICI & First time & Lacunar infarction of bilateral basal ganglia \\
\hline 3 & Male & 59 & AICI & First time & $\begin{array}{l}\text { Multiple lacunar infarction of bilateral cerebellar hemisphere, medulla, frontal, } \\
\text { temporal and parietal lobe, basal ganglia and corona radiata }\end{array}$ \\
\hline 4 & Male & 70 & AICI & Second time & Left pons softening focus, left cerebellar hemispheres infarction \\
\hline 5 & Male & 60 & AICI & First time & Lacunar infarction of back side of pons and bilateral basal ganglia \\
\hline 6 & Male & 53 & AICI & Second time & $\begin{array}{l}\text { Multiple lacunar infarction of bilateral basal ganglia, centrum semiovale on the } \\
\text { right side }\end{array}$ \\
\hline 7 & Male & 62 & AICI & First time & $\begin{array}{l}\text { Left pons softening focus, left cerebellar hemisphere infarction and lacunar } \\
\text { infarction of bilateral basal ganglia }\end{array}$ \\
\hline 8 & Female & 71 & $\begin{array}{l}\text { Nasopharyngeal Carcinoma post } \\
\text { Radiotherapy (NCR) }\end{array}$ & First time & Radiation encephalopathy \\
\hline 9 & Female & 40 & NCR & First time & Radiation encephalopathy \\
\hline 10 & Male & 52 & AICI & First time & Multiple lacunar infarction of right side of medulla, corona radiata \\
\hline 11 & Male & 54 & Motor neuron disease & First time & $\mathrm{N} / \mathrm{A}$ \\
\hline 12 & Male & 58 & Tongue cancer post glossectomy & First time & N/A \\
\hline
\end{tabular}

Table 1: Basic demographic and clinical information for the 12 patients with swallowing disorders. 
3. The Distance of Laryngeal Elevation (DLE) [24,25]. The reference point of laryngeal movement is the posterior-superior corner of the subglottic air column. The start of laryngeal elevation begins with the video frame showing the first upward movement and ends with the video frame showing the last downward movement. A 50 cents coin was affixed in the anterior neck region of each subject. Adjustment quotient was calculated by measurement $(\mathrm{cm})$ of the diameter of the coin in the video divided by the actual measurement $(\mathrm{cm})$ of the diameter of the coin. The actual measurement of the laryngeal elevation was calculated by the measurement of the laryngeal elevation in the video multiply the adjustment quotient. The normal value is $2 \mathrm{~cm}$ or more

\section{Contact of Tongue Base to Pharyngeal Wall (CTP)}

5. Cricopharyngeal Muscle Achalasia (CMA). The presence of CMA is judged in three scenarios. a). In patients who showed significant PSR, if reduced laryngeal elevation or inadequate downward pressure can be ruled out, then CMA was judged to be present. Inadequate downward pressure was judged present if there was no contact between tongue base and posterior pharyngeal wall as observed in VFSS or reduced pharyngeal contraction as observed in FEES $[20,21,25]$. b). If PSR was significant, but either laryngeal elevation or reduced downward pressure was observed, then the presence of CMA was based on the presence of cricopharyngeal bar when the sphincter was open without associated CP relaxation during VFSS [26], or presence of esophageal strictures during FEES [16]. c). If incomplete laryngeal elevation or/and inadequate downward pressure of the bolus is observed, and cricopharyngeal bar or esopharyngeal stricture was not observed during swallowing, then CMA is indicated as "unable to judge" (NTJ).

A total of 6 variables were included in the study. PSR and WPC were derived from FEES and VFSS, and PDT, DLE, CMA, CTP were derived from VFSS studies. Even though PSR was measured subjectively in our study, measurements from both FEES and VFSS have been highly consistent. All variables in pre-treatment were reported in table 2.

\begin{tabular}{|c|c|c|c|c|c|c|}
\hline Patient No. & PSR (\%) & PDT (s) & DLE (cm) & CMA & WPC & CTP \\
\hline 1 & $90 \%$ & 1.7 & 3.4 & Yes & No & Yes \\
\hline 2 & $80 \%$ & 0.3 & 4.3 & Yes & No & Yes \\
\hline 3 & $100 \%$ & 0 & 2.8 & Yes & No & Yes \\
\hline 4 & $60 \%$ & 2.31 & 0.5 & UTJ & Yes & Yes \\
\hline 5 & $50 \%$ & 0 & 1.5 & UTJ & Yes & Yes \\
\hline 6 & $100 \%$ & 0 & 4.3 & Yes & No & Yes \\
\hline 7 & $50 \%$ & 0 & 1 & UTJ & Yes & Yes \\
\hline 8 & $50 \%$ & 0 & 1.3 & Yes & Yes & No \\
\hline 9 & $90 \%$ & 0 & 0.6 & Yes & Yes & No \\
\hline 10 & $100 \%$ & 0 & 1 & UTJ & Yes & Yes \\
\hline 11 & $100 \%$ & 0 & 1 & Yes & No & Yes \\
\hline 12 & $80 \%$ & 0 & 0.8 & Yes & Yes & No \\
\hline
\end{tabular}

Table 2: Six swallowing variables in pre-treatment.

PSR: Pyriform Sinus Residue; PDT: Pharyngeal Delay Time; DLE: Distance of Laryngeal Elevation; CMA: Cricopharyngeal Muscle Achalasia; WPC: Weak Pharyngeal Contraction; CTP: Contact Between Tongue Base and Posterior Pharyngeal Wall

\section{Treatment}

Balloon dilatation was initiated within 1 week of PSR confirmation in all 12 patients. A 14FR balloon catheter (total length $39 \mathrm{~cm}$ ) was inserted into the esophagus through the patient's mouth; Firstly, the catheter was placed into the oropharynx, then further inserted downward into laryngopharynx while the patient was allowed to swallow the catheter till most of which entered the esophagus through the cricopharyngeus, leaving $8 \mathrm{~cm}$ length of the catheter out of mouth, then patient was asked to sustain /a/ sound. The presence of normal voice ensured the catheter was in the esophagus, not in the airway. Next, an initial volume of $4 \mathrm{ml}$ of purified water was injected into the catheter balloon slowly, the injector was removed, and the catheter was pulled slowly until it exited the mouth. The volume of injected water was gradually increased ( 1 additional $\mathrm{ml}$ of water consecutively, up to a total of $10 \mathrm{ml}$ ). The session of balloon dilatation lasted for 30 minutes, repeating eight dilatations per session, three sessions per week, for six sessions over a 2-week period.

\section{Statistical analysis}

The pre-and post-treatment VFSS and FEES results of the 12 patients were collected, recorded, and analyzed. Continuous variables PSR, PDT, and DLE were analyzed using paired t-tests. Discrete variables, CMA and WPC were analyzed using nonparametric Wilcoxon signed-ranks test. All data collection and analysis were performed using SPSS 21.0 (IBM SPSS Statistics 21, SPSS Inc., USA).

\section{Intra-rater and inter-rater agreement}

Two rehabilitation physicians (PW and SJX) and a speech-language pathologist (MWY) analyzed the digital VFSS files. The 24 videos (12 pre-treatment, 12 post-treatment) were randomly numbered without patient identification information. Each rater analyzed the 24 videos twice at least 72 hours apart to minimize recall bias. Similarly, the digital FEES files of the 12 patients were independently reviewed by an otolaryngologist (XHC), an otolaryngology resident (YBY) and a rehabilitation physician (PW) on two separate occasions separated by at least 72 hours. Twenty percent of the total measurements for CMA and WPC were randomly selected to calculate inter-and intra-rater reliability. The kappa coefficient was used to assess the intra-and inter-rater reliability and a value of 0.7 and above indicated high intra-and inter-rater reliability. Intra-observer agreement was 0.88 and 0.92 , and inter-observer agreement was 0.82 and 0.85 for CMA and WPC.

Intraclass correlations were used to analyze intra-and inter-observer agreement for continuous variables PSR, PDT and DLE. Intra-observer agreement was $0.92,0.95,0.92$, and inter-observer agreement was $0.87,0.91,0.88$ for PSR, PDT, DLE.

\section{Results}

The post treatment data is reported in table 3 . Among the 12 patients who received $\mathrm{BD}$, eight patients $(66.67 \%)$ made marked progress with FOIS levels reached 5 to 7 and were able to resume oral intake $(66.67 \%)$ while four patients (No. 4, No. 7, No. 8, No. 12) showed limited improvements with FOIS remained at level 1 to 3 and were unable to discontinue tube feeding. The patients in our study were contacted 6 month and one year after the study regarding their swallow function to track the long-term effect of $\mathrm{BD}$. The 8 patients who showed significant improvement post BD continued to enjoy the benefits of $\mathrm{BD}$ and remained on oral diet. The 4 patients who did not 
benefit from BD continued to show poor swallow function and were remained on tube feeding for nutrition purpose.

\begin{tabular}{|c|c|c|c|c|c|c|}
\hline Patient No. & PSR & PDT & DLE & CMA & WPC & CTP \\
\hline 1 & $15 \%$ & 0 & 2 & No & No & Yes \\
\hline 2 & $20 \%$ & 0.33 & 2 & No & No & Yes \\
\hline 3 & $20 \%$ & 0 & 0.7 & No & No & Yes \\
\hline 4 & $80 \%$ & 0 & 1.5 & UTJ & No & Yes \\
\hline 5 & $5 \%$ & 0 & 1.5 & No & No & Yes \\
\hline 6 & $10 \%$ & 0 & 1.8 & No & No & Yes \\
\hline 7 & $90 \%$ & 0 & 1.0 & UTJ & No & Yes \\
\hline 8 & $60 \%$ & 0.66 & 1.23 & No & No & No \\
\hline 9 & $30 \%$ & 0 & 0.6 & No & No & No \\
\hline 10 & $0 \%$ & 0 & 1.5 & No & No & Yes \\
\hline 11 & $0 \%$ & 0 & 1 & No & No & Yes \\
\hline 12 & $50 \%$ & 0 & 1 & UTJ & Yes & No \\
\hline
\end{tabular}

Table 3: Six swallowing variables in post treatment.

PSR: Pyriform Sinus Residue; PDT: Pharyngeal Delay Time; DLE: The Distance of Laryngeal Elevation; CMA: Cricopharyngeal Muscle Achalasia; WPC: Weak Pharyngeal Contraction; CTP: Contact between Tongue Base and Posterior Pharyngeal wall; UTJ: Unable to Judge

\section{Changes in PSR, PDT and DLE post balloon dilatation}

Paired t-tests were used to compare the differences in the following variables: PSR, PDT, DLE pre-and post-BD. Table 4 shows that there was a significant difference in PSR $(\mathrm{p}=0.002)$ while the difference between pre-and post-treatment was not significant for PDT $(p=0.275)$ or DLE $(p=0.110)$. Our study found incomplete laryngeal elevation was not associated with improvement post-BD in 4 patients $(\mathrm{p}=0.11)$. In patients No.3, No.7, No.9, No.11, less than $2 \mathrm{~cm}$ of the hyoid elevation was found both pre-and post-BD, even though their PSR has shown improvement.

\begin{tabular}{|c|c|c|c|c|c|}
\hline & Pre-Treat & Post-Treat & Correlation (p) & T value & p (2-tailed) \\
\hline PSR & $0.75 \pm 0.22$ & $0.29 \pm 0.30$ & $-0.443(0.113)$ & 3.814 & 0.002 \\
\hline PDT & $0.31 \pm 0.73$ & $0.07 \pm 0.19$ & $-0.113(0.701)$ & 1.140 & 0.275 \\
\hline DLE & $1.76 \pm 1.346$ & $1.25 \pm 0.47$ & $0.645(0.013)$ & 1.714 & 0.110 \\
\hline
\end{tabular}

Table 4: Comparison of the three continuous variables pre-versus post-balloon dilatation $(\mathrm{n}=12)$.

PSR: Pyriform Sinus Residue; PDT: Pharyngeal Delay Time; DLE: The Distance of Laryngeal Elevation

\section{Changes in CMA and WPC post balloon dilatation}

Table 5 shows that there was a significant reduction in CMA between pre-and post-BD values $(\mathrm{p}=0.004)$, indicating that $\mathrm{BD}$ eliminated CMA. Comparing table 3 and 4 , the current study showed that all identified CMA was eliminated post BD. There was also a significant difference in WPC $(\mathrm{p}=0.008)$ between pre-and post-BD. Even though one patient continued to show WPC post-BD, as a group there was a significant reduction in WPC $(\mathrm{p}=0.008)$ between pre-and postBD.

\section{Discussion}

$\mathrm{BD}$ is a common procedure used to treat $\mathrm{CPD}$ and pharyngo-upper esophageal strictures in dysphagia patients $[2,6,10,14,15]$. There have not been any studies investigated the effectiveness of BD in dysphagia management from the perspective of swallow physiology changes. Our current study is the only study that investigates the effectiveness of BD in reduction of PSR using instrumentation techniques to identify swallow physiology change in dysphagia patients. The two most well used instrumentation techniques, VFSS and FEES, were used to identify CMA, WPC, PDT and DLE, and we observed that CMA and WPC were most closely related to PSR. BD was effective in eliminating PSR in 8 out of 12 cases. In these 8 effective cases, five cases have shown CMA in various medical diagnoses. Four patients were diagnosed with infarction of the basal ganglia and/or corona radiate and one with motor neuron disease. All five cases involved upper motor damages which may be the reason for CMA. BD worked in all five cases, suggesting that BD contributes to improve UES relaxation and eliminate PSR.

Of the eight effective cases, three cases have shown WPC. Two patients were diagnosed with brain stem infarction and one patient was diagnosed with nasopharyngeal cancer post radiation. In our study, we found BD improved WPC, this result is consistent with the findings by Stokely et al., who postulated that interventions targeted on improving pharyngeal contraction should be effective in reducing post-swallow residue [27]. Lan et al., has proposed that BD served as a safe sensory stimulation to induce active resistance exercise in swallowing muscles, which in turn improved the pharyngeal driving force, further promoting UES relaxation and emptying pyriform sinus [4]. The proposed mechanism may explain the effect of balloon dilation on improvement of WPC in our study.

The four patients that did not respond to BD were all due to anatomical and physiological changes of swallow mechanism resulted from their medical diagnoses. Based on the clinical symptoms of left peripheral facial paralysis, deafness and tinnitus, limited left jaw opening, dizziness, dysphagia, limited jaw movement as well MRI results, patients No. 4 and 7 were diagnosed with infarction of the left pontocerebellar trigone with involvement of pontocerebellar fibers. The pontocerebellar trigone lies at the intersection of the pons, medulla, and cerebellum [28]. The cortex-pons-cerebellum-cortex circle is one of the important extrapyramidal systems, playing a role in the regulation of muscle tone, coordination of muscle activity, and habitual motions such as swallowing [28]. Therefore, it was hypothesized that the infarct in these patients resulted in discoordination of swallowing subsystems such as laryngeal elevation, tongue base retraction, pharyngeal contraction and cricopharyngeal muscle opening.

\begin{tabular}{|c|c|c|c|c|c|c|}
\hline \multirow{2}{*}{ Pretreat-Posttreat } & \multicolumn{4}{|c|}{ Ranks } & \multicolumn{2}{|c|}{ Test Statistics } \\
\hline & Type of ranks based & $\mathbf{N}$ & Mean rank & Sum of ranks & $\mathbf{z}$ & p (exact test, (2-tailed) \\
\hline CMA & Negative ranks & $2^{\mathrm{a}}$ & 6.77 & 74.50 & -2.863 & 0.004 \\
\hline WPC & Positive ranks & $0^{\mathrm{b}}$ & 0.00 & 0.00 & -2.646 & 0.008 \\
\hline
\end{tabular}

Table 5: Wilcoxon signed ranks test of discrete variables pre-versus post-balloon dilatation $(\mathrm{n}=12)$.

a, posttreatment $<$ pretreatment; $b$, posttreatment $>$ pretreating

CMA: Cricopharyngeal Muscle Achalasia; WPC: Weak Pharyngeal Contraction 
Patient no. 8 with a diagnosis of radiotherapy post nasopharyngeal carcinoma and patient no. 12 with a diagnosis of partial glossectomy post oral cancer shared some similarities in their swallow physiology changes and their treatment outcomes. Both of the patients showed CMA and WPC in their pretreatment assessment. Both lost large bulk of tongue tissue in their cancer treatment, for patient no. 8 it was due to severe atrophy of the tongue including the Base of Tongue (BOT), and for patient no. 12 it was due to removal of more than $50 \%$ of the tongue. Past research has shown reduced bulk of tongue can result in more severe dysphagia. Patients with less than 50\% tongue resection scored significantly higher in water swallow test than those with more than $50 \%$ resection [29]. It is worth to mention patient no. 9, who was diagnosed with nasopharyngeal carcinoma but with no atrophy of the tongue, showed good improvement post BD. This finding suggested that tongue volume might be a key factor in promoting deglutition. O'Connell et al., [9] have reported that reconstructive surgery that preserved the bulk of the Base of the Tongue (BOT) after cancer treatment helped maintain adequate BOT-PPW (Posterior Pharyngeal Wall) contact, which generated the necessary force to propel the food bolus through the oropharynx.

Our study found incomplete laryngeal elevation was not associated with improvement post-BD in 4 patients. It is well known that Laryngeal Elevation (LE) contributes to closure of the airway entrance, and the forward movement of the larynx contributes to opening of the upper esophageal sphincter [23]. In this study, we did not find a correlation of DLE and reduced PSR. This was consistent with the results reported by Kraaijenga et al., [30] no cohort correlations between residue ratings and hyoid displacement were found in their study. PSR in our study might be explained by other, non-hyoid, mechanical variables.

In this study, CMA was diagnosed indirectly through VFSS and FEES. To assess CMA more accurately, further research using electrophysiological approach such as electromyographyis necessary [31]. PSR was measured subjectively in our current study, utilizing the normalized residue ratio scale would provide an objective way of measuring residue in the pyriform sinus [32]. This study used pre-and post-treatment design, which cannot completely exclude the possibility of spontaneous recovery. Future research utilizing a combination of electromyography, VFSS and FEES to assess CMA or WPC would further elucidate the pathomechanisms of PSR, exploring the relation between CMA and WPC in order to ensure the action of BD, and to improve treatment decision-making.

\section{Conclusion}

The current study found that patients with the swallow physiology change of CMA or WPC result from various medical diagnoses include cerebral vascular accidents, motor neuron disease and head and neck cancer benefit significantly from BD to reduced residue in the pyriform sinuses therefore improving pharyngeal dysphagia. BD has no effect on PSR resulted from discoordination of swallowing subsystems in infarction of the pontocerebellar trigone or losing large bulk of tongue tissue in case of cancer treatment. These findings can help clinical decision making in choosing the most appropriate candidates for $\mathrm{BD}$.

\section{Acknowledgement}

This study was funded in part by the project of clinical instruction of rehabilitation of TCM (Grant No. ZY3-LCPT-1-1008) and in part by research program of educational committee in Shanghai "Research of neuropathic mechanism about pyriform sinus residue after acute ischemic brain infarction" (Grant No. A1-163010182).

We are grateful to the following people for their help and guidance in this study: Mr. David Nolfi from Duqense University, Professor Jason $\mathrm{Hu}$ from the School of Rehabilitation Medicine at Shanghai University of Traditional Chinese Medicine (TCM); Professor Zaoming Huang from the Key Laboratory of Speech \& Hearing Sciences of the Ministry of Education; Dr Jianning Zhang from the ENT Department of Affiliated Yueyang Hospital, Shanghai University of TCM; Dr Yunyun Zhang from the Department of Neurology of affiliated Yueyang Hospital, Shanghai University of TCM; Dr Yi Wang from the Department of Electromyography of affiliated Yueyang Hospital, Shanghai University of TCM; and Dr Lequn Zhu from the Department of Radiology of affiliated Yueyang Hospital, Shanghai University of TCM.

\section{References}

1. Park WY, Lee TH, Ham NS, Park JW, Lee YG, Cho et al. (2015) Adding Endoscopist-Directed Flexible Endoscopic Evaluation of Swallowing to the Videofluoroscopic Swallowing Study Increased the Detection Rates of Penetration, Aspiration, and Pharyngeal Residue. Gut Liver 9: 623628.

2. Dou Z, Wan G, Wang X (2016) Two case reports of Balloon dilatation treating cricopharyngeal achalasia. Chin J Phys Med Rehabil 28: 166170.

3. Perlman A, Schulze-Delrieu K (2003) Deglutition and Its Disorders: Anatomy, Physiology, Clinical Diagnosis, and Management $\left(6^{\text {th }}\right.$ edn $)$, Delmar/Cengage Learning, New York, USA.

4. Wan P, Chen X, Zhu L, Xu S, Huang L, et al. (2016) Dysphagia Post Subcortical and Supratentorial Stroke. J Stroke Cerebrovasc Dis 25: 7482 .

5. Bian RX, Choi IS, Kim JH, Han JY, Lee SG (2009) Impaired opening of the upper esophageal sphincter in patients with medullary infarctions. Dysphagia 24: 238-245.

6. Lan Y, Xu G, Dou Z, Wan G, Yu F, et al. (2013) Biomechanical changes in the pharynx and upper esophageal sphincter after modified balloon dilatation in brainstem stroke patients with dysphagia. Neurogastroenterol Motil 25: 821-829.

7. Agarwal J, Palwe V, Dutta D, Gupta T, Laskar SG, et al. (2011) Objective assessment of swallowing function after definitive concurrent (chemo) radiotherapy in patients with head and neck cancer. Dysphagia 26: 399406.

8. Halczy-Kowalik L, Wiktor A, Rzewuska A, Kowalczyk R, Wysocki R, et al. (2015) Compensatory Mechanisms in Patients After a Partial or Total Glossectomy due to Oral Cancer. Dysphagia 30: 738-750.

9. O'Connell DA, Rieger J, Harris JR, Dziegielewski P, Zalmanowitz J, et al. (2008) Swallowing function in patients with base of tongue cancers treated with primary surgery and reconstructed with a modified radial forearm free flap. Arch Otolaryngol Head Neck Surg 134: 857-864.

10. Francis DO, Hall E, Dang JH, Vlacich GR, Netterville JL, et al. (2015) Outcomes of serial dilation for high-grade radiation-related esophageal strictures in head and neck cancer patients. Laryngoscope 125: 856-862.

11. Ku PKM, Vlantis AC, Leung SF, Lee KY, Cheung DM, et al. (2010) Laryngopharyngeal sensory deficits and impaired pharyngeal motor function predict aspiration in patients irradiated for nasopharyngeal carcinoma. The Laryngoscope 120: 223-228.

12. Estanislao N, Flores HB, Ang E, Salvana AD, Tripon ES, et al. (2009) Comparison of the efficacy of rigid dilators and balloon dilators for benign esophageal strictures-a meta-analysis. Journal of Gastroenterology \& Hepatology 24: 87-87. 
13. Kelly EA, Koszewski IJ, Jaradeh SS, Merati AL, Blumin JH, et al. (2014) Botulinum Toxin Injection for the Treatment of Upper Esophageal Sphincter Dysfunction. Annals of Otology. Ann Otol Rhinol Laryngol 122: 100-108.

14. Oh TH (2014) Treatments with balloon catheter dilatation and botulium toxin injection in a patient with pharyngeal dysphagia secondary to cricopharyngeal dysfunction. Korean J Gastroenterol 63: 325-328.

15. Maejima R, Ijijima K, Koike T, Ara N, Uno K, et al. (2015) Endoscopic balloon dilatation for pharyngo-upper esophageal stricture after treatment of head and neck cancer. Dig Endosc 27: 310-316.

16. Hu Y, Wei X, Dou Z (2011) Mechanism of balloon dilation treating cricopharyngeal dysfunction. Chin J Brain Dis Rehabil Electron Ed 1: 82-87.

17. Eisenhuber E, Schima W, Schober E, Pokieser P, Stadler A, et al. (2002) Videofluoroscopic assessment of patients with dysphagia: pharyngeal retention is a predictive factor for aspiration. AJR Am J Roentgenol 178 393-398.

18. Fiebach JB, Schellinger PD, Jansen O, Meyer M, Wilde P, et al. (2002) $\mathrm{CT}$ and diffusion-weighted MR imaging in randomized order: diffusion-weighted imaging results in higher accuracy and lower interrater variability in the diagnosis of hyperacute ischemic stroke. Stroke 33: 2206-2210.

19. Crary MA, Mann GDC, Groher ME (2005) Initial psychometric assessment of a functional oral intake scale for dysphagia in stroke patients. Arch Phys Med Rehabil 86: 1516-1520.

20. Fuller SC, Leonard R, Aminpour S, Belafsky PC (2009) Validation of the pharyngeal squeeze maneuver. Otolaryngol Head Neck Surg 140: 391-394.

21. Rodriguez KH, Roth CR, Rees CJ, Belafsky PC (2007) Reliability of the pharyngeal squeeze maneuver. Ann Otol Rhinol Laryngol 116: 399-401.

22. Zhu ZH, Zhou F, Xiao F (2009) Study of barium dynamic imaging of pharynx in patients with deglutition disorder and normal swallowing. Mod Med Health.
23. Logemann JA (1993) Manual for the Videofluorographic Study of Swallowing $\left(2^{\text {nd }}\right.$ edn), Pro-Ed, Texas, USA.

24. Logemann JA, Pauloski BR, Rademaker AW, Kahrilas PJ (2002) Oropharyngeal swallow in younger and older women: videofluoroscopic analysis. J Speech Lang Hear Res 45: 434-445.

25. Logemann JA (1997) Evaluation and Treatment of Swallowing Disorders, $\left(2^{\text {nd }} e d n\right)$, Pro-Ed, Texas, USA.

26. Sivarao DV, Goyal RK (2000) Functional anatomy and physiology of the upper esophageal sphincter. Am J Med 108: 27-37.

27. Stokely SL, Peladeau-Pigeon M, Leigh C, Molfenter SM, Steele CM (2015) The Relationship Between Pharyngeal Constriction and Post-swallow Residue. Dysphagia 30: 349-356.

28. Zhu C (2009) Neuroanatomy, ( $2^{\text {nd }}$ edn), People's Medical Publishing House, Connecticut, USA.

29. Zhang L, Huang Z, Wu H, Chen W, Huang Z (2014) Effect of swallowing training on dysphagia and depression in postoperative tongue cancer patients. European Journal of Oncology Nursing 18: 626-629.

30. Kraaijenga SA, van der Molen L, Heemsbergen WD, Remmerswaal GB, Hilgers FJ, et al. (2017) Hyoid bone displacement as parameter for swallowing impairment in patients treated for advanced head and neck cancer. Eur Arch Otorhinolaryngol 274: 597-606.

31. Alfonsi E, Merlo IM, Ponzio M, Montomoli C, Tassorelli C, et al. (2010) An electrophysiological approach to the diagnosis of neurogenic dysphagia: implications for botulinum toxin treatment. J Neurol Neurosurg Psychiatry 81: 54-60.

32. Pearson WG Jr, Molfenter SM, Smith ZM, Steele CM (2013) Image-based measurement of post-swallow residue: the normalized residue ratio scale. Dysphagia 28: 167-177. 